\title{
Enteroviral Infection Leads to Transactive Response DNA-Binding Protein 43 Pathology in Vivo
}

Yuan Chao Xue, ${ }^{* \dagger}$ Chelsea M. Ruller, ${ }^{\ddagger}$ Gabriel Fung, ${ }^{* \dagger}$ Yasir Mohamud, ${ }^{* \dagger}$ Haoyu Deng, ${ }^{* \dagger}$ Huitao Liu, ${ }^{* \Uparrow}$ Jingchun Zhang, ${ }^{* \dagger}$ Ralph Feuer, ${ }^{\ddagger}$ and Honglin Luo ${ }^{* \dagger}$

From the Centre for Heart Lung Innovation, * and the Departments of Pathology and Laboratory Medicine ${ }^{\dagger}$ and Experimental Medicine, ${ }^{\natural}$ University of British Columbia, Vancouver, British Columbia, Canada; the Integrated Regenerative Research Institute at San Diego State University, ${ }^{\ddagger}$ Department of Biology, San Diego State University, San Diego, California; and the Department of Vascular Surgery, ${ }^{\S}$ Renji Hospital, School of Medicine, Shanghai Jiaotong University, Shanghai, China

\author{
Accepted for publication \\ August 30, 2018. \\ Address correspondence to \\ Ralph Feuer, Ph.D., The Inte- \\ grated Regenerative Research \\ Institute (IRRI) at San Diego \\ State University, Cell \& \\ Molecular Biology Joint \\ Doctoral Program, Department \\ of Biology, San Diego State \\ University, 5500 Campanile \\ Drive, San Diego, CA 92182- \\ 4614; or Honglin Luo, M.Sc., \\ M.D., Centre for Heart Lung \\ Innovation, St. Paul's Hospital, \\ Room 166, 1081 Burrard Street, \\ Vancouver, BC, Canada V6Z \\ 1Y6. E-mail: rfeuer@sdsu.edu \\ or honglin.luo@hli.ubc.ca.
}

\begin{abstract}
Amyotrophic lateral sclerosis (ALS) is a fatal neurodegenerative disease that primarily affects motor neurons in the cerebral cortex, brainstem, and spinal cord, leading to progressive paralysis and eventual death. Approximately $95 \%$ of all ALS cases are sporadic without known causes. Enteroviruses have been suspected to play a role in ALS because of their ability to target motor neurons and to cause muscle weakness and paralysis. In vitro enteroviral infection results in cytoplasmic aggregation and cleavage of transactive response DNA binding protein-43, a pathologic hallmark of ALS. However, whether enteroviral infection can induce ALS-like pathologies in vivo remains to be characterized. In this study, neonatal BALB/C mice were intracranially inoculated with either a recombinant coxsackievirus B3 expressing enhanced green fluorescent protein or mock-infected for 2, 5, 10, 30, and 90 days. Histologic and immunohistochemical analysis of brain tissues demonstrated sustained inflammation (microglia and astrogliosis) and lesions in multiple regions of the brain (hippocampus, cerebral cortex, striatum, olfactory bulb, and putamen) in parallel with virus detection as early as 2 days for up to 90 days after infection. Most notably, ALS-like pathologies, including cytoplasmic mislocalization of transactive response DNA binding protein-43, p62-, and ubiquitin-positive inclusions, were observed in the areas of infection. These data provide the first pathologic evidence to support a possible link between enteroviral infection and ALS. (Am J Pathol 2018, 188: 2853-2862; https://doi.org/10.1016/ j.ajpath.2018.08.013)
\end{abstract}

Amyotrophic lateral sclerosis (ALS) is a neurodegenerative disease that primarily targets motor neurons of the central nervous system (CNS), but it may also result in secondary effects, such as muscle weakness and cognitive impairment. ${ }^{1-3}$ The prevalence of ALS in both Europe and North America is around three to five cases per 100,000 individuals each year, with the overall lifetime risk increasing with age and being higher in men than in women (1:350 versus 1:400). ${ }^{1,3}$ Currently, there is no cure for ALS. Two treatments approved by the Food and Drug Administration can only modestly delay disease progression and prolong the lifespan of the patients. ${ }^{4}$

ALS is typically grouped into two categories: familial and sporadic ALS. Familial ALS is responsible for approximately $5 \%$ of all cases and is associated with mutations in $>30$ genes that encode proteins involved in various cellular functions, including vesicle trafficking, RNA processing, and axonal integrity. ${ }^{5,6}$ Sporadic ALS, however, accounts for the majority of ALS cases (approximately 95\%)

\footnotetext{
Supported by the Canadian Institutes of Health Research grant PJT159546 (H.L.), ALS Canada (H.L.), and the Natural Sciences and Engineering Research Council grant RGPIN-2016-03811 (H.Lu.). Y.M. is also the recipient of ALS Canada-Brain Canada Doctoral Fellowship. Y.C.X. is also supported by UBC ECOSCOPE training program. Y.C.X., Y.M., and H.D. are the recipients of a four-year Ph.D. fellowship from the University of British Columbia.

Disclosures: None declared.
} 
without clear cause. A gene-environment interaction model that involves both genetic mutations and environmental risk factors, such as viral exposure, physical activity, smoking, heavy metals, and pesticides and chemicals, has been proposed to contribute to the development and progression of sporadic ALS.

Two widely studied viruses in the context of ALS are human endogenous retrovirus (HERV) and enterovirus (EV). Increased gene expression of HERV-K and reverse transcriptase activity have been found in the blood and brain samples of ALS patients. ${ }^{8}$ Moreover, transgenic mouse study reveals that expression of HERV-K in the neurons can lead to motor dysfunction, suggesting a role of HERV infection in ALS. ${ }^{9}$ However, despite great efforts for the discovery of EV genome in ALS samples, the clinical data are controversial, ${ }^{10-16}$ probably because of the differences in viral detection techniques and/or in the disease stage of sample collection, as well as the potential virus-triggered prion-like mechanism, in which an active viral infection may not be required for ALS progression. ${ }^{17}$

EVs are a family of positive, single-stranded RNA viruses, which include poliovirus, coxsackievirus, echovirus, and EV (eg, EV-A71 and EV-D68). ${ }^{17}$ EVs are known to infect the CNS and are responsible for various neurologic diseases, such as poliomyelitis, meningitis, encephalitis, and non-polio flaccid paralysis. ${ }^{18,19}$ In addition to acute infection, EVs can also cause a persistent infection within the CNS, which may be reactivated years later. ${ }^{20-22}$ Although a causal relationship between chronic EV infection and ALS development remains to be established, our previous research in cultured cells demonstrates that EV infection induces ALS-like transactive response DNA-binding protein-43 (TDP-43) pathology, that is, cytoplasmic mislocalization, aggregation, and cleavage of TDP- $43,{ }^{23}$ indicating a possible involvement of EVs in ALS pathogenesis.

Here, we investigated the neuropathologic phenotype of a persistent CNS infection of coxsackievirus B3 (CVB3) in mice. Our results reveal that EV infection induces a similar ALS-like pathology in vivo.

\section{Materials and Methods}

\section{Isolation and Production of Recombinant Coxsackievirus}

Recombinant CVB3 expressing enhanced green fluorescent protein (eGFP-CVB3) was generated as described previously. ${ }^{24}$ In brief, eGFP (Clonetech, Palo Alto, CA) was inserted into pMKS1, a plasmid that includes a unique SfiI restriction enzyme site in the backbone of CVB3 clone $\mathrm{pH} 3$. The new pMKS1 was transfected into COS cells for 48 hours, and the cell culture was subjected to three cycles of freeze-thaw on dry ice to release viruses. After centrifugation, the supernatant was harvested and added to HeLa RW cell culture. After 48 hours, eGFP-CVB3 virus was collected and plaque purified.

\section{Mouse Breeding and Viral Inoculation}

BALB/c mice were obtained from Harlan Sprague Dawley (Harlan Laboratories, San Diego, CA). The breeding pairs were monitored daily to identify pups within 24 hours of birth. The neonates at 2 to 3 days old were intracranially inoculated with either a nonlethal dose of eGFP-CVB3 $\left(1 \times 10^{6}\right.$ plague-forming units) or equal volume $(25 \mu \mathrm{L})$ of Dulbecco's modified Eagle's medium (mock infection) as previously reported. ${ }^{20}$ All mice survived except for a few who died from the procedure of intracranial injection, independent of viral infection. All animal procedures were approved by the San Diego State University Institutional Animal Care and Use Committee and are in strict accordance with the Public Health Service Policy and United States Department of Agriculture Animal Welfare Regulations.

\section{Mouse Brain Preparation}

At 2, 5, 10, 30, and 90 days after infection, mice were euthanized in a bell jar with isoflurane, followed by immediate decapitation. Mouse brains were harvested and fixed with $4 \%$ formaldehyde.

\section{Immunohistochemistry and Histochemical Staining}

Paraffin-embedded sections (4- $\mu \mathrm{m}$ thick) were deparaffinized first through xylene and then with a gradually decreasing concentration of isopropanol $(100 \%, 90 \%$, and $70 \%$ ). Hematoxylin and eosin staining was conducted to evaluate virus-induced brain damage. The sections were also subjected to immunohistochemical (IHC) staining. Briefly, antigen retrieval was performed by heating the sections in citrate buffer pH 6.0 (005000; Life Technologies Carlsbad, CA) for 25 minutes at $121^{\circ} \mathrm{C}$. Slides then underwent peroxidase blocking by using hydrogen peroxide $(30 \mathrm{mg} / \mathrm{mL})$, followed by washes with $1 \times$ tris-buffered saline $(0.05 \mathrm{~mol} / \mathrm{L}$ Tris, $0.155 \mathrm{~mol} / \mathrm{L} \mathrm{NaCl}, \mathrm{pH} 7.6)$. After blocking, sections were incubated with primary antibodies overnight at $4^{\circ} \mathrm{C}$. The MACH4 Universal HRP-Polymer Detection System (BRI4012H; Biocare Medical, Pacheco CA) was then used to detect the staining according to the manufacture's procedure. At the end of the procedure, all slides were also counterstained with hematoxylin solution Gill II (GHS232; Sigma-Aldrich, St. Louis, MO).

Mouse brain tissues were immunostained by using the follow primary antibodies diluted in tris-buffered saline/ phosphate-buffered saline ( $1 \%$ bovine serum albumin, $1.5 \mathrm{~mol} / \mathrm{L} ; \mathrm{NaCl}, 0.5 \mathrm{~mol} / \mathrm{L} ; \mathrm{pH}$ 7.6): double-stranded RNA (dilution 1:1000; J2 mouse IgG2a monoclonal antibody; 10010200; Scicon, Szirák, Hungary), GFP (dilution 1:200; mouse monoclonal antibody; sc-9996; Santa Cruz Biotechnology, Santa Cruz, Dallas, TX), glial fibrillary acidic protein (GFAP; dilution 1:200; mouse monoclonal antibody; SMC-441; StressMarq, Victoria, BC, Canada), ionized calcium binding adaptor molecule 1 (Iba1; dilution 
1:200; mouse monoclonal antibody; sc-32725; Santa Cruz Biotechnology), pSTAT3 (dilution 1:200; mouse monoclonal antibody; sc-8059; Santa Cruz Biotechnology), choline acetyltransferase (ChAT; dilution 1:500; mouse monoclonal antibody; sc-55557; Santa Cruz Biotechnology), RZ3 (pThr-231 Tau; dilution 1:300; mouse monoclonal antibody; courtesy of Dr. Peter Davies at the Feinstein Institute for Medical Research, New York, NY), TDP-43 (dilution 1:1000; rabbit polyclonal antibody; 10782-2-AP; Proteintech, Rosemont, IL), p62/ sequestosome-1 (SQSTM1; dilution 1:200; mouse monoclonal antibody; sc-28359; Santa Cruz Biotechnology), and ubiquitin (dilution 1:2000; mouse monoclonal antibody; sc-8017; Santa Cruz Biotechnology).

Images were taken by using the Aperio ScanScope AT (Digital slide scanner; Leica Biosystems Inc., Buffalo Grove, IL) or a Nikon Eclipse E600 microscope equipped with a SPOT Flex Model 15.2 64 Mp Shifting Pixel camera (Diagnostic Instrument Inc., Sterling Heights, MI).

\section{Quantification and Statistical Analysis}

Quantification of immunohistochemistry images was performed by using ImageJ version 1.0 (NIH, Bethesda, MD) with the combination of Color Deconvolution Plugin version 1.5 (http://www.mecourse.com/landinig/software/ cdeconv/cdeconv. html) to generate the optical density value based on the intensity of the staining as described. ${ }^{25}$ The quantified results are presented as means \pm SD for two separate brain regions (hippocampus and cerebral cortex) at the indicated time points after infection. Statistical analysis was conducted by one-way analysis of variance (ANOVA), followed by Bonferroni's multiple comparison test by using GraphPad Prism software version 6.0c (GraphPad Software, La Jolla, CA). $P$ value of 0.05 was considered to be statistically significant.

\section{Results}

Establishment of a Persistent Viral Infection within the Mouse Brain

To determine the in vivo impacts of $\mathrm{EV}$ infection on the neuropathology, the neonates of $\mathrm{BALB} / \mathrm{c}$ mice, a EV-susceptible strain, were intracranially inoculated with a nonlethal dose of eGFP-CVB3 or mock-infected with Dulbecco's modified Eagle's medium for 2, 5, 10, 30, or 90 days. Mouse brains were harvested for viral detection by IHC staining by using antibodies against GFP and doublestranded RNA, an intermediate form of the replicating viral genomic RNA. IHC staining of GFP (virally expressed protein) demonstrated focal infections located mainly in the hippocampus region, with the most active infection occurring between day 2 and day 5 post-infection (PI) (Figure 1). The GFP levels gradually decreased but remained detectable up to day 90 PI. The IHC signal for double-stranded RNA peaked at day 5 PI and then markedly reduced with only faint detection on days 30 and 90 PI. These results were consistent with the previous reports that viral RNA that peaked at day 2 to 5 PI can still be detected by real-time RT-PCR up to day $90 \mathrm{PI},{ }^{26}$ suggesting a persistent viral infection within the brain. In addition to the hippocampus, viral infections in other areas of the brain, that is, cerebral cortex (especially the neocortex) (Figure 1), olfactory bulb, striatum, and putamen (data not shown), were also observed. Corresponding to the peak infection time and areas, histologic examination by hematoxylin and eosin staining revealed massive immune cell infiltrations and substantial tissue damages between day 2 and day 10 PI within the regions of viral infection (Figure 2). Tissue vacuolization was observed at the later times (Figure 2). These results supported previous findings that CVB3 has a high tendency to infect actively replicating cells that usually reside within the neurogenic regions, such as the hippocampus, olfactory bulb, and lateral ventral regions of the CNS to establish persistent viral infections. ${ }^{27-29}$

\section{Increased CNS Immune Responses after Infection}

Because neuroinflammation plays an important role in ALS pathogenesis, ${ }^{30}$ the effects of EV exposure on the pattern and extent of brain inflammation were studied. IHC staining was conducted to assess the level of astrocyte reactivity with anti-GFAP and anti-pSTAT3 antibodies, microglial activation using anti-Ibal antibody, and natural killer (NK) cell maturation and neurogenesis with anti-ChAT antibody.

Compared with mock infection, CVB3-infected mice at various time points after infection demonstrated increased immune responses. In the hippocampus region, IHC staining of GFAP, a reactive astrocyte marker, ${ }^{31}$ displayed significant increases in expression intensity at day 5 to 10 and day 90 PI (Figure 3, A and B). Likewise, another reactive astrocyte marker, pSTAT $3,{ }^{32}$ also exhibited enhanced CNS astrocyte activation, in particular at day 5 PI. As a marker of microglia, ${ }^{33}$ Iba1 staining revealed a continuous immune response (microgliosis) toward the viral infection with a peak expression at day 5 PI. IHC staining for ChAT, a marker for NK cell maturation and neurogenesis, ${ }^{34}$ demonstrated a strong reaction at the early phase of viral infection, and the immunoreactivity remained detectable until the end of the experiment. Similarly, significantly increased GFAP staining was observed at day 5 and day $90 \mathrm{PI}$ in the cerebral cortex region (Figure 3C). Together, these data indicated that CVB3 induced microgliosis, astrogliosis, and activation of NK cells in persistently infected mouse brains.

\section{ALS-Related Proteinopathies after Intracranial CVB3 Infection}

Familial ALS and sporadic ALS share the pathologic feature of TDP-43, which has been identified in $>95 \%$ of all ALS cases. $^{35,36}$ Mice with chronic CVB3 infection exhibited 

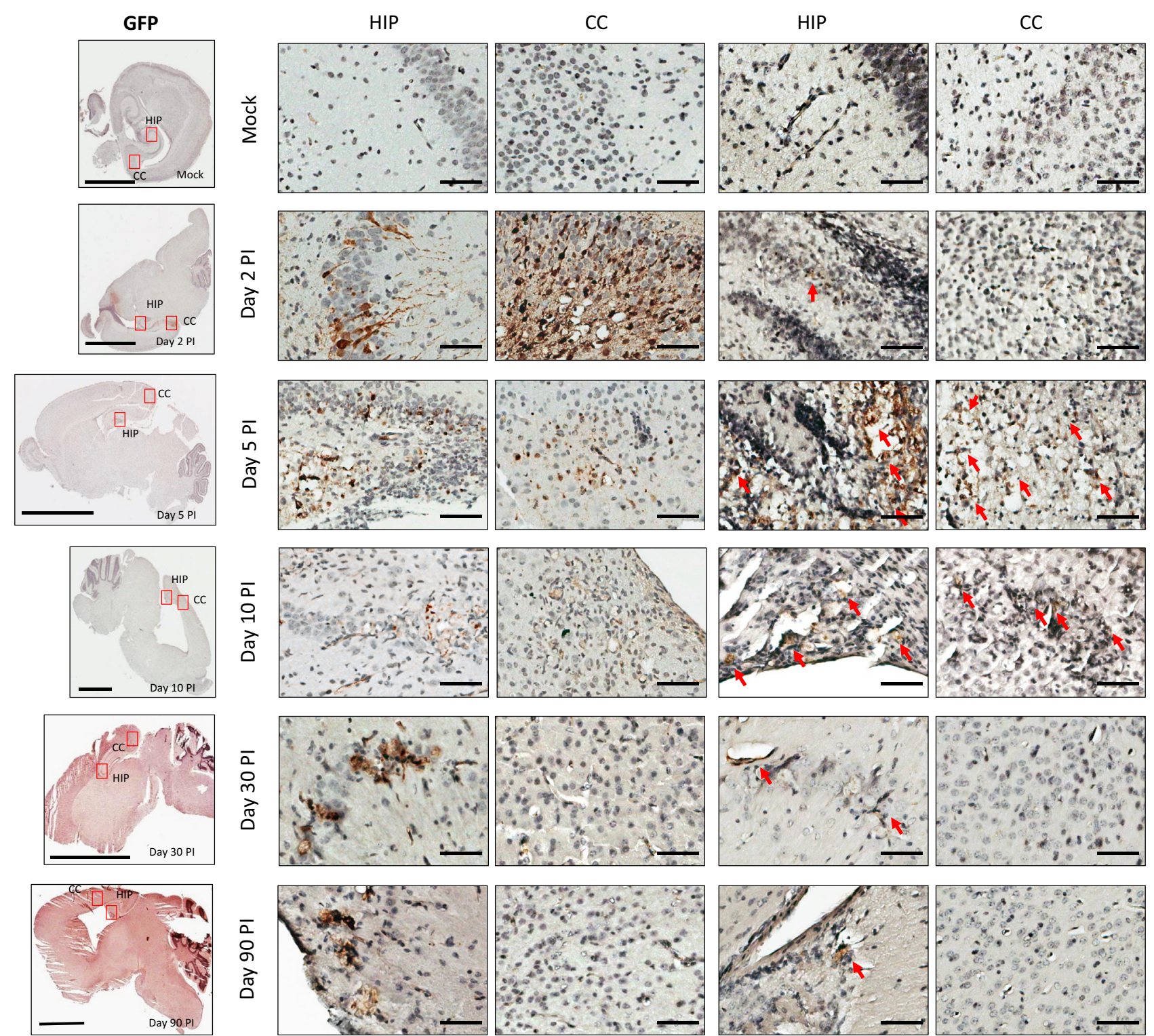

Figure 1 A nonlethal dose of coxsackievirus B3 (CVB3) causes a persistent viral infection in mouse brain. Two- to 3-day-old BALB/C neonatal mice were either infected intracranially with a nonlethal dose of enhanced green fluorescent protein (eGFP)-CVB3 $\left(1 \times 10^{6}\right.$ plaque-forming unit) or mock-infected with Dulbecco's modified Eagle's medium. Mouse brains were harvested at day 2, 5, 10, 30, and 90 post-injection (PI). Immunohistochemical staining with an anti-GFP or anti-double-stranded RNA (dsRNA) antibody (brown) was performed, followed by nuclear counterstaining with hematoxylin. The figure includes the whole-slide view (left column; anti-GFP staining) to illustrate the distribution of the infection and the corresponding enlarged images of the infected regions (all other columns). The red arrows indicate dsRNA-positive staining as focal infections in the tissue. $n=3$ individual mouse brain samples at each time point. Scale bars: $2 \mathrm{~mm}$ (left column, GFP mock, GFP Day 10 PI); 3 mm (left column, GFP Day 2 PI, GFP Day 5 PI, GFP Day 90 PI); 5 mm (left column, GFP Day $30 \mathrm{PI}) ; 60 \mu \mathrm{m}$ (all other columns). CC, cerebral cortex; HIP, hippocampus.

signs of flaccid tail tone and hind limb dysfunction. ${ }^{20} \mathrm{CVB} 3$ infection resulted in cytoplasmic mislocalization and aggregation of TDP-43 in virus-infected HeLa cells ${ }^{23}$ and primarily isolated mouse neuronal cells (Y.C.X. et al., unpublished data). Here, we extended our previous work to examine the distribution and expression pattern of TDP-43 in CVB3-infected mouse brains. ${ }^{23}$ To better correlate virus-infected areas with TDP-43 alteration, two serial slides from mock and CVB3-infected mouse brains were stained for GFP and TDP-43, respectively. TDP-43 was predominantly localized in the nucleus in mock-infected neuronal cells, whereas in day 5 CVB3-infected brain within the hippocampus region, TDP-43 was mostly mislocalized to the cytoplasm, along with nuclear down-regulation (Figure 4A). Notably, at days 30 and 90 PI, TDP-43 pathology remained apparent in the survival cells within the infected areas (Figure 4B), suggesting a similar pathologic phenotype to ALS. 

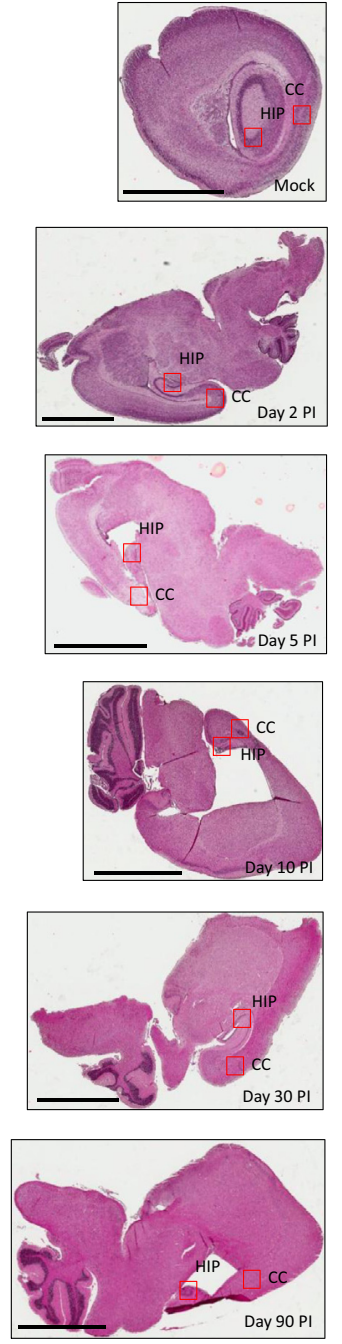

HIP
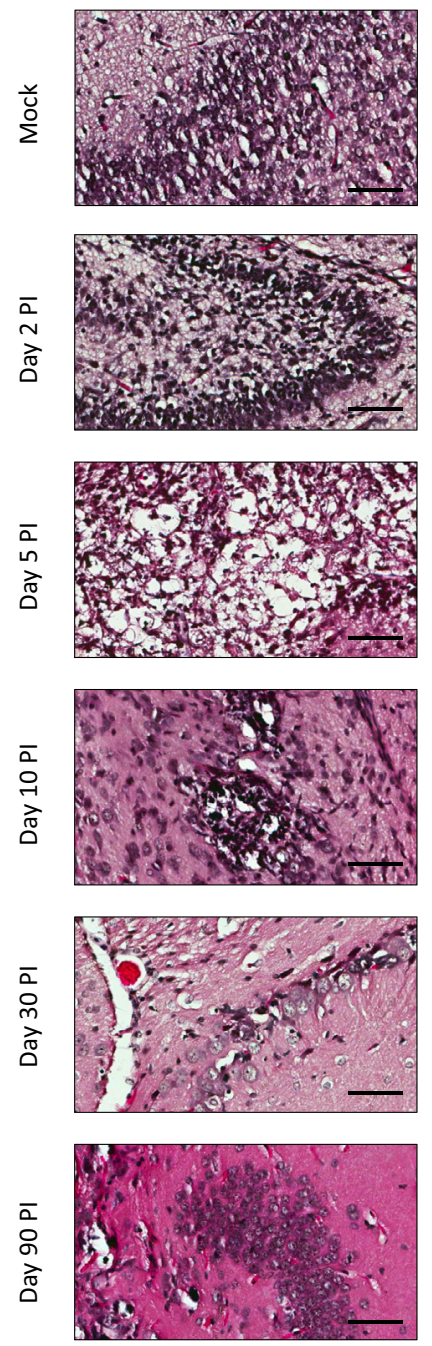

CC
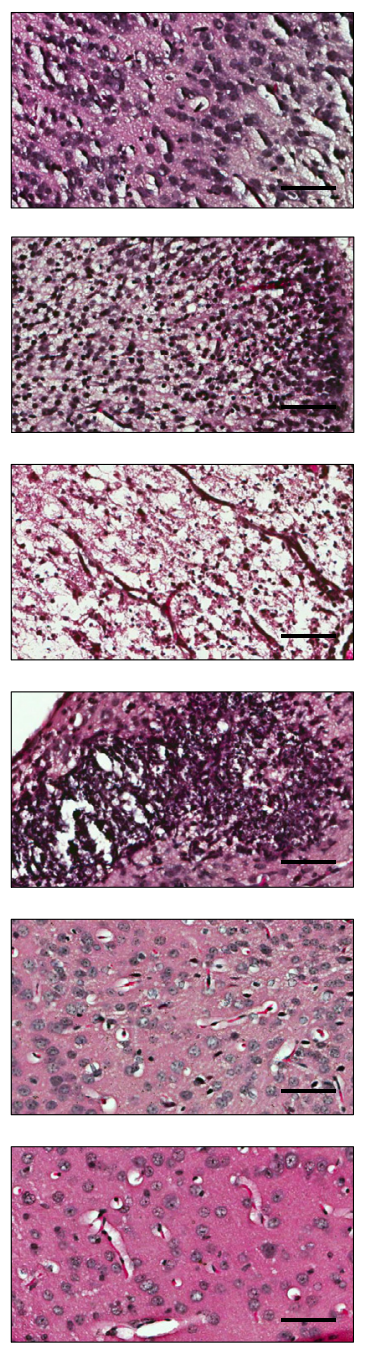

Figure 2 Intracranial coxsackievirus B3 infection results in brain lesions. Hematoxylin and eosin staining was conducted on the collected mouse brain tissues. The left column of the whole tissue images illustrate the distribution of viral infection, and the middle and right columns show the corresponding magnified images of the infected regions. Within the area of viral infection, immune cell infiltration and tissue lesions can be observed. $n=3$ individual mouse brain tissues at each time point post-infection (PI). Scale bars: $2 \mathrm{~mm}$ (left column, mock and Day 2 PI); 3 mm (left column, Day 5 PI, Day 10 PI, Day $30 \mathrm{PI}$, Day $90 \mathrm{PI}$ ); $60 \mu \mathrm{m}$ (middle and right columns). CC, cerebral cortex; HIP, hippocampus.
To further characterize the cytoplasmic inclusions, brain tissues were stained for p62/SQSTM1 (a ubiquitin-binding protein), ubiquitin, and phosphorylated Tau (a microtubule-associated protein). Both p62 and ubiquitin were associated with protein aggregates detected in ALS. ${ }^{37}$ Within the similar infected regions observed in TDP-43 pathology, p62/SQSTM1 and ubiquitin were stained positive at all infection time points, with the exception of the mock and the faintly positive day 2 PI samples (Figure 5). However, the immunoreactivity of phosphorylated Tau, which has been observed in ALS with cognitive impairment, ${ }^{38}$ was not evident within the infected region until day 90 PI (Figure 5).

\section{Discussion}

To properly associate EV infection with the pathology of ALS in infected mouse brains, the areas of viral infection were confirmed, and ALS-associated pathologic phenotypes within the identified regions of infection were analyzed. CVB3 infections were focally localized in multiple areas of the brain, including the hippocampus and cerebral cortex, and caused massive tissue damages, that is, structural fragmentation and vacuolization at early and late infections, respectively. This pattern of infection is likely because of the susceptibility of migrating neuronal progenitor cells to CVB3 infection and/or the distribution of viral receptors (ie, coxsackievirus and adenovirus receptor, and its co-receptor decay-accelerating factor). ${ }^{20,27,28}$ Virusmediated damages of the hippocampus could lead to possible behavioral changes, such as poor long-term memory and spatial navigation, whereas impairments to the cerebral cortex, especially the neocortex (the newest evolutionary layer of cerebral cortex), might affect cognition, sensory perception, and motor function. ${ }^{39}$ Overall, the various possible consequences of $\mathrm{EV}$ infection appear to correlate with the symptoms associated with several neurodegenerative diseases, such as ALS and Alzheimer 
A
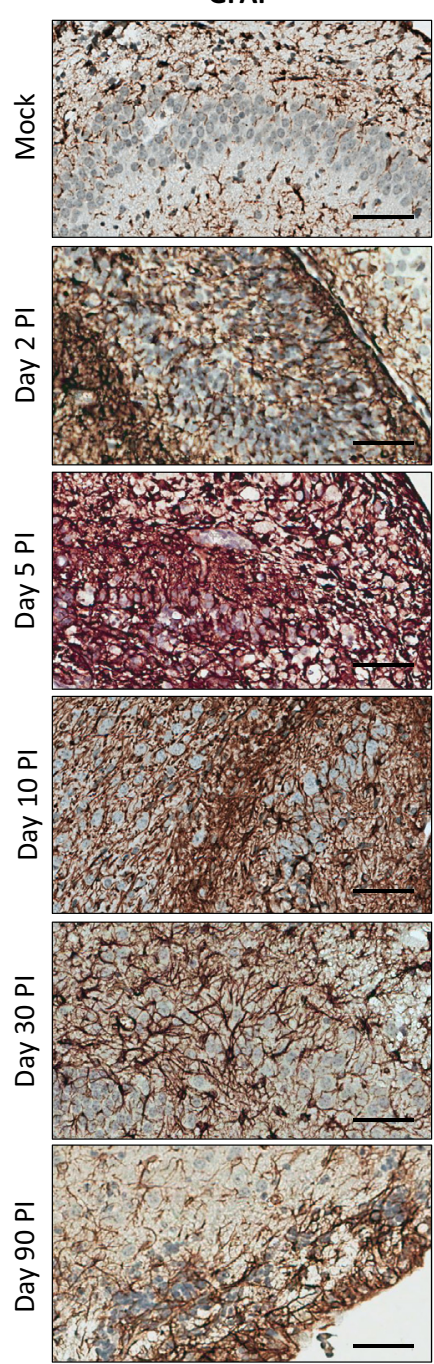

B

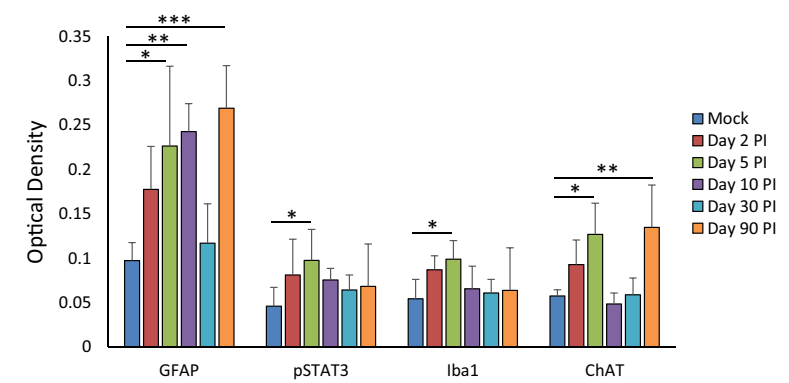

PSTAT3
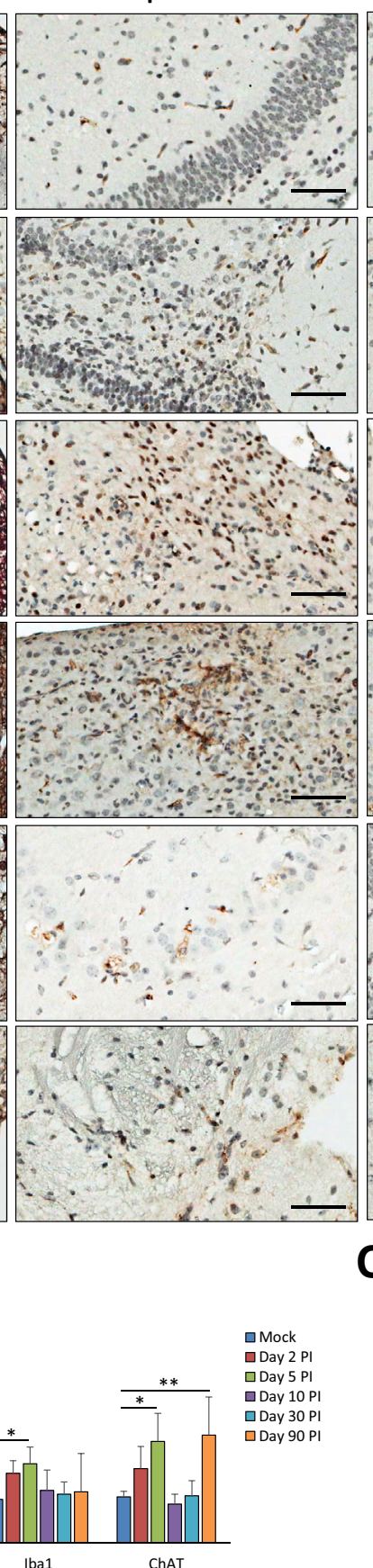
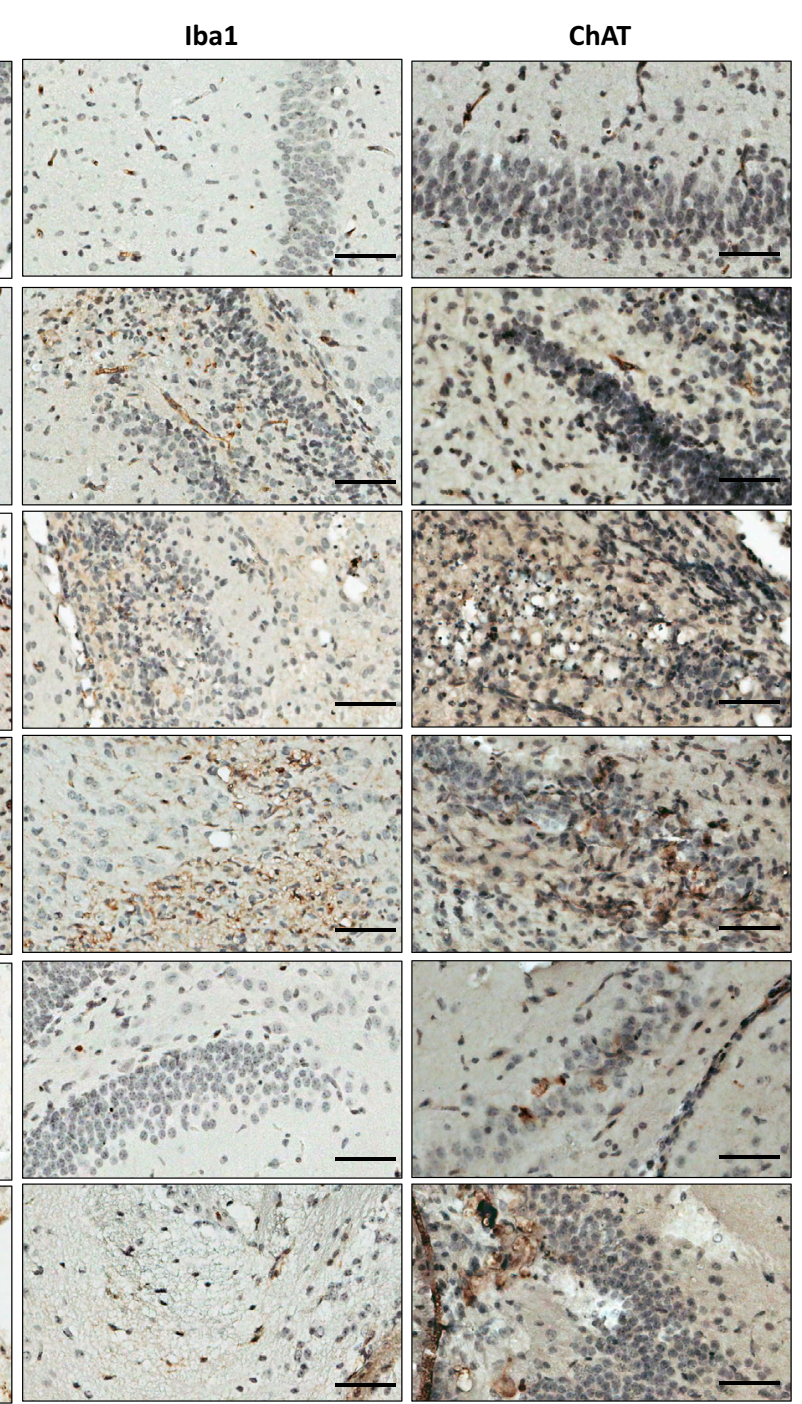

C ${ }^{0.35} \stackrel{* * *}{* *^{*}}$

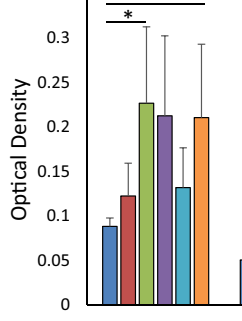

GFAP

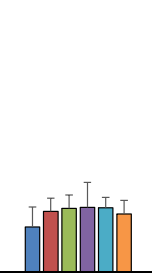

PSTAT3

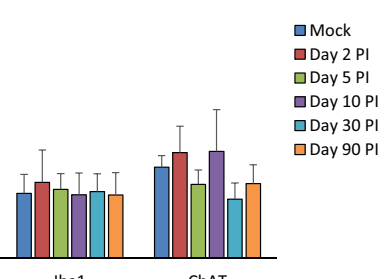

Figure 3 Coxsackievirus B3 (CVB3) infection caused increased immune responses in the mouse brain. A: Mouse brain tissues were harvested, and immunohistochemical staining was performed with antibodies against glial fibrillary acidic protein (GFAP), pSTAT3, ionized calcium binding adaptor molecule 1 (Iba1), and choline acetyltransferase (ChAT) as indicted. B and C: CVB3-induced immune responses in the hippocampus (B) or cerebral cortex (C) region were quantified by optical density as described in the Materials and Methods. Data are expressed as means \pm SD. $n=3$ to 6 individual mouse brain samples in the hippocampus region at each time point (A); $n=5$ mock samples (B and $\mathbf{C}) ; n=3$ samples at day 2 post-infection (PI) (B and $\mathbf{C}) ; n=4$ samples at day 5 PI (B and $\mathbf{C}) ; n=4$ samples at day $10 \mathrm{PI}$ (B and $\mathbf{C}) ; n=6$ samples at day $30 \mathrm{PI}(\mathbf{B}$ and $\mathbf{C}) ; n=6$ samples at day $90 \mathrm{PI}$ (B and $\mathbf{C})$. Statistical analysis was performed by one-way analysis of variance (ANOVA), followed by Bonferroni's multiple comparison test. ${ }^{*} P<0.05,{ }^{*} P<0.01$, and ${ }^{* * *} P<0.001$. Scale bars $=60 \mu \mathrm{m}$.

disease. Even though motor dysfunction was seen during the experimentation, more precise and robust behaviors and physiological tests, however, will be required to confidently link EV infection with clinical signs of neurodegenerative diseases.
Within the infected regions, levels of GFAP and pSTAT3, which mark the activation of reactive astrocytes, were markedly increased with a peak at 5 to 10 days and remained high until 90 days PI. This result indicates an elevated reactive astrocyte response. In addition, an 
A
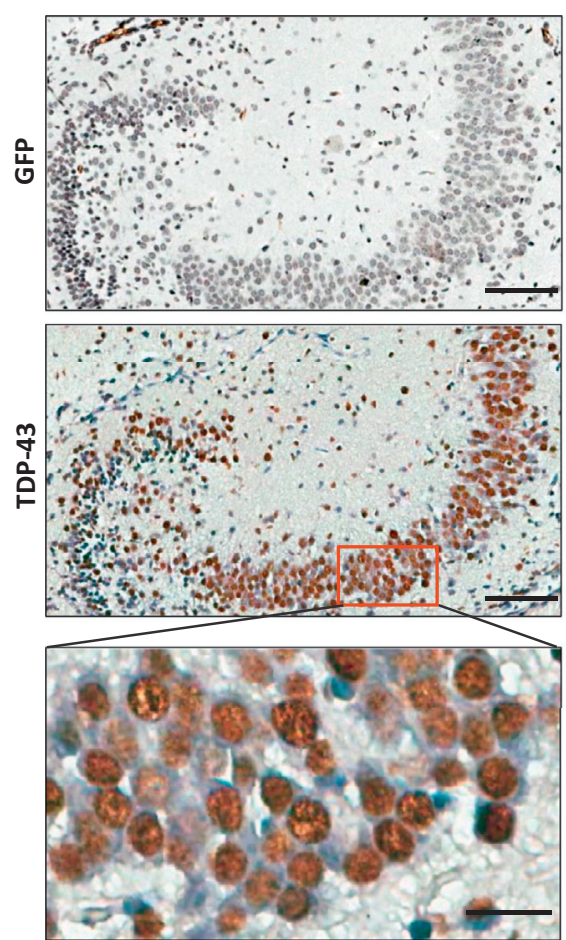

Day 5 PI
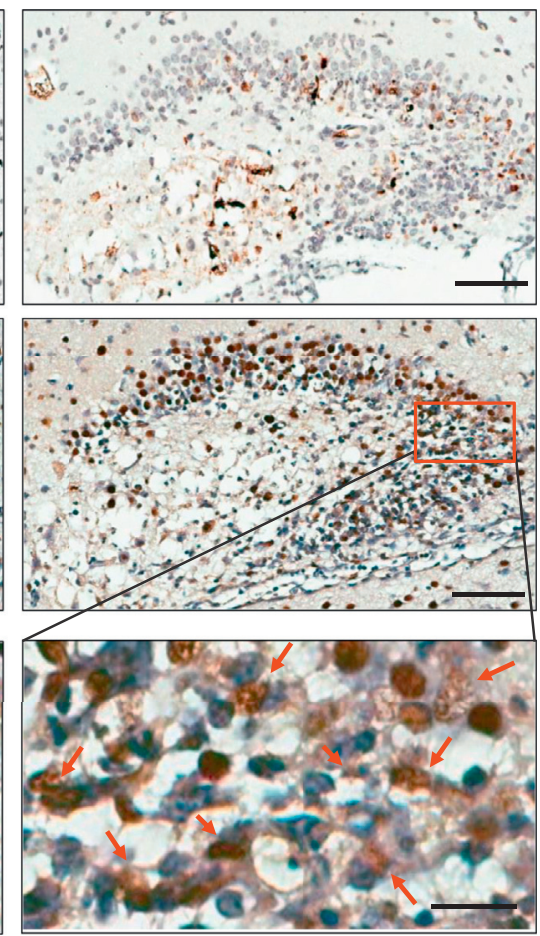

B
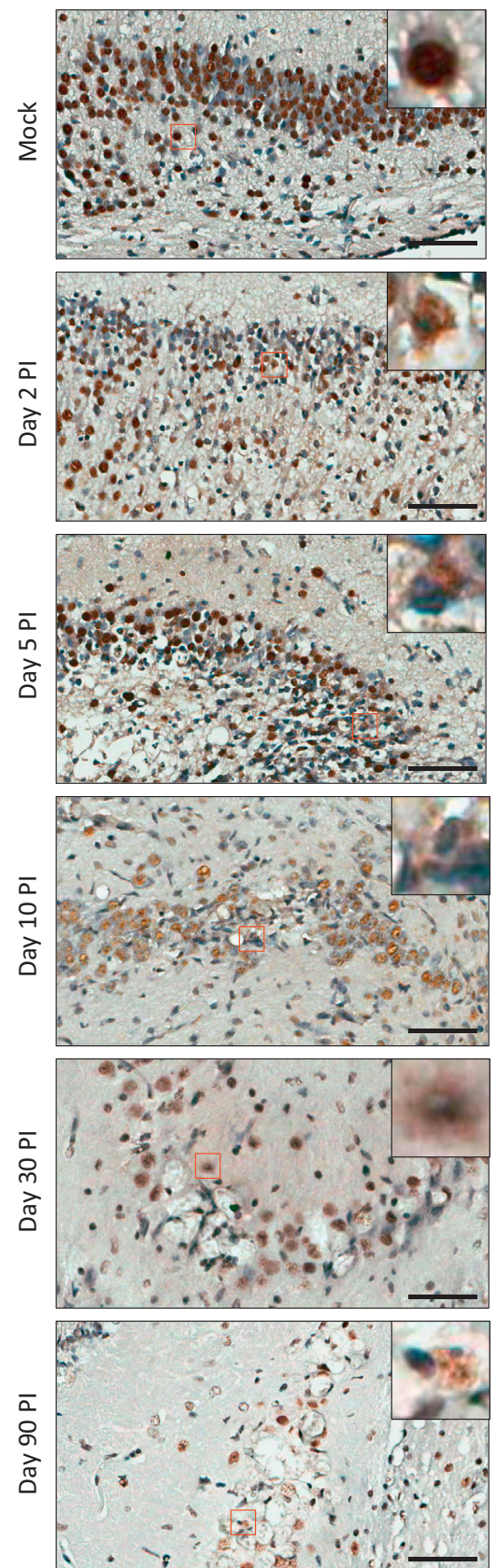

Figure 4 Coxsackievirus B3 infection led to cytoplasmic mislocalization of transactive response DNA binding protein-43 (TDP-43) in the brain. Immunohistochemical staining on mouse brains was conducted as above by using anti-green fluorescent protein (GFP) and anti-TDP-43 antibodies. A: Representative images of TDP-43 staining in the region of enhanced (e)GFP-CVB3-infected hippocampus at day 5 post-infection (PI). Red boxes indicate the location of the enlarged images; red arrows, to the observed TDP-43 cytoplasmic mislocalization. B: Immunohistochemical staining images of TDP-43 in the hippocampus region at different time points after infection. Boxed areas are shown at higher magnification in the insets. $n=3$ individual mouse brain samples at each time point. Scale bars: $60 \mu \mathrm{m}$ (A, top and middle row, and B); $30 \mu \mathrm{m}$ (A, bottom row).

increase in infiltration/activation of microglia/macrophage within the infected regions, in particular the hippocampus region at early time points PI, was also observed through Iba1 immunostaining. Collectively, these data suggest a peak immune response toward an acute virus infection in the brain, and then a sustained low level of microglia/ macrophage response to a chronic infection that is likely led by autoreactive $\mathrm{T}$ cells. ${ }^{40}$ Of more interest, elevated stimulations were also observed in both NK cell maturation and neurogenesis at day 5 and day 90 PI, specifically in the hippocampus region, by ChAT staining. Activation of microglia/macrophages in this context could stimulate circulating NK cells and excite the release of cytokines and chemokines, such as IL-15 and IL-12, which are coincidentally elevated in both the serum and cerebral spinal fluid from EV-infected patients and ALS patients. ${ }^{19,41}$ Building on previous findings that active immune systems are likely the promoters for ALS-like neurodegeneration, ${ }^{1-3}$ we speculate that in addition to virus-induced direct damages to the CNS, injury secondary to host immune responses may drive the development of the disease even further. 


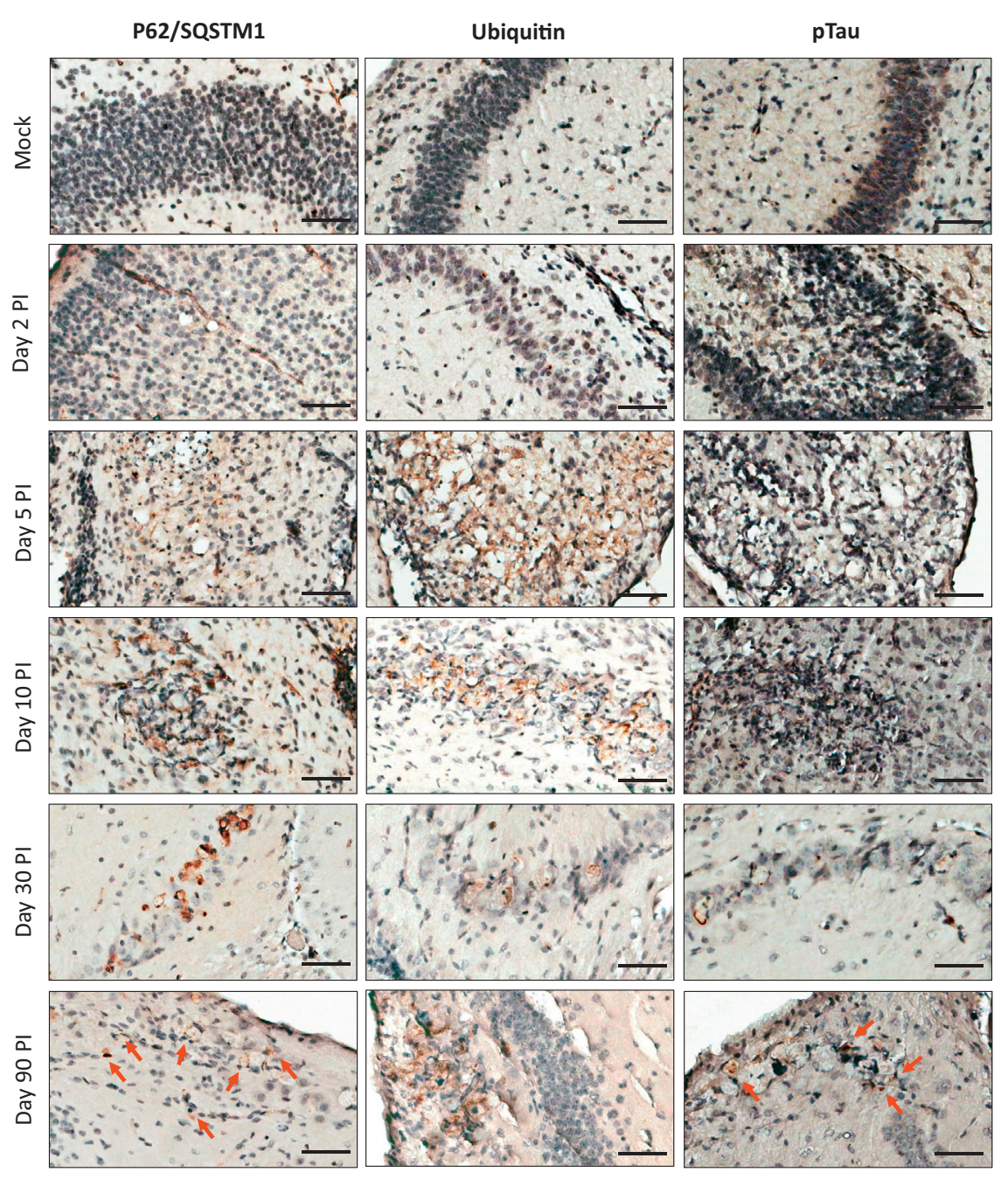

Figure 5 Coxsackievirus B3 infection induced the accumulation of p62- and ubiquitin-positive inclusions in the brain. Hippocampus regions were immunostained by using antibodies against p62/sequestosome-1 (SQSTM1), ubiquitin, and phosphorylated Tau (pTau) as indicated. Red arrows point to the positive staining within the illustrated tissues. $n=3$ individual mouse brain tissues at each time point. Scale bars $=60 \mu \mathrm{m}$. PI, post-infection.

Intracellular protein inclusions are a pathologic hallmark of ALS neurodegeneration. TDP-43 inclusion is particularly important because it is identified in most ALS cases, contributing significantly to the pathogenesis of ALS. $^{42}$ Within EV-infected regions, typical TDP-43 pathology in the form of nuclear-to-cytoplasmic mislocalization could be seen. The degree/frequency of TDP-43 mislocalization appeared to be correlated well with the severity of the infection, in which day $5 \mathrm{PI}$, the peak of viral infection, has a larger area of TDP-43 abnormalities than later time points. This is likely because virus-infected cells die as a result of productive viral replication after the acute viremia period. However, the remaining survival cells in the infected areas retained abnormal TDP-43 phenotype. In addition to TDP-43, $\mathrm{EV}$-infected regions showed strong positive staining for p62/SQSTM1 and ubiquitin, two proteins also known as major components of ALS-related inclusions. ${ }^{37}$ Of interest, phosphorylation of tau protein was observed only at day 90 PI. Phosphorylated tau is not generally associated with ALS except for individuals with impaired cognition. $^{38}$ The mechanisms by which EV infection induces ALS-like proteinopathies are unclear. Expression of CVB3-encoded proteinase $2 \mathrm{~A}$ causes cytoplasmic redistribution of TDP-43, ${ }^{23}$ suggesting a mechanism of EV-induced TDP-43 pathology through the disruption of nucleocytoplasmic trafficking by viral proteinase cleavage of nucleoporin proteins. ${ }^{43}$ Moreover, recent findings that EVs, including CVB3, poliovirus, and EV-D68, inhibit autophagic flux by preventing the formation of the soluble N-ethylmaleimide-sensitive factor activating protein receptor complex indicate that enhanced accumulation of p62 and ubiquitin may be a consequence of defective autophagy. $^{44,45}$

\section{Conclusions}

The present study demonstrates that CVB3 infection induces characteristics of TDP-43 pathology in virus-infected neural cells, accompanied by the presence of CNS lesions, microgliosis, and astrogliosis in persistently infected mouse 
brains. Even though human studies had failed to establish a conclusive relationship between ALS and EV infection, ${ }^{17}$ animal models, unlike human models in which many external and internal factors cannot be controlled, will confirm the likelihood of this connection in a more controlled manner. Therefore, although animal studies like the one presented here may not lead to a solid conclusion, they can prompt more robust and specific clinical studies in the causational relationship between ALS patients and EV infection in which a more decisive result could be discovered.

\section{Acknowledgment}

We thank Dr. Peter Davies (Feinstein Institute for Medical Research, New York, NY) for generously providing the RZ3 (pThr-231 Tau) monoclonal antibody.

\section{Supplemental Data}

Supplemental material for this article can be found at https://doi.org/10.1016/j.ajpath.2018.08.013.

\section{References}

1. Brown RH Jr, Al-Chalabi A: Amyotrophic lateral sclerosis. N Engl J Med 2017, 377:1602

2. Hardiman O, Al-Chalabi A, Chio A, Corr EM, Logroscino G, Robberecht W, Shaw PJ, Simmons Z, van den Berg LH: Amyotrophic lateral sclerosis. Nat Rev Dis Primers 2017, 3:17085

3. van Es MA, Hardiman O, Chio A, Al-Chalabi A, Pasterkamp RJ, Veldink JH, van den Berg LH: Amyotrophic lateral sclerosis. Lancet 2017, 390:2084-2098

4. Martinez A, Palomo Ruiz MD, Perez DI, Gil C: Drugs in clinical development for the treatment of amyotrophic lateral sclerosis. Expert Opin Investig Drugs 2017, 26:403-414

5. Al-Chalabi A, van den Berg LH, Veldink J: Gene discovery in amyotrophic lateral sclerosis: implications for clinical management. Nat Rev Neurol 2017, 13:96-104

6. Chia R, Chio A, Traynor BJ: Novel genes associated with amyotrophic lateral sclerosis: diagnostic and clinical implications. Lancet Neurol 2018, 17:94-102

7. Al-Chalabi A, Calvo A, Chio A, Colville S, Ellis CM, Hardiman $\mathrm{O}$, Heverin M, Howard RS, Huisman MH, Keren N, Leigh PN, Mazzini L, Mora G, Orrell RW, Rooney J, Scott KM, Scotton WJ, Seelen M, Shaw CE, Sidle KS, Swingler R, Tsuda M, Veldink JH, Visser AE, van den Berg LH, Pearce N: Analysis of amyotrophic lateral sclerosis as a multistep process: a population-based modelling study. Lancet Neurol 2014, 13:1108-1113

8. Kury P, Nath A, Creange A, Dolei A, Marche P, Gold J, Giovannoni G, Hartung HP, Perron H: Human endogenous retroviruses in neurological diseases. Trends Mol Med 2018, 24:379-394

9. Li W, Lee MH, Henderson L, Tyagi R, Bachani M, Steiner J, Campanac E, Hoffman DA, von Geldern G, Johnson K, Maric D, Morris HD, Lentz M, Pak K, Mammen A, Ostrow L, Rothstein J, Nath A: Human endogenous retrovirus-K contributes to motor neuron disease. Sci Transl Med 2015, 7:307ra153

10. Berger MM, Kopp N, Vital C, Redl B, Aymard M, Lina B: Detection and cellular localization of enterovirus RNA sequences in spinal cord of patients with ALS. Neurology 2000, 54:20-25
11. Giraud P, Beaulieux F, Ono S, Shimizu N, Chazot G, Lina B: Detection of enteroviral sequences from frozen spinal cord samples of Japanese ALS patients. Neurology 2001, 56:1777-1778

12. Nix WA, Berger MM, Oberste MS, Brooks BR, McKenna-Yasek DM, Brown RH Jr, Roos RP, Pallansch MA: Failure to detect enterovirus in the spinal cord of ALS patients using a sensitive RT-PCR method. Neurology 2004, 62:1372-1377

13. Swanson NR, Fox SA, Mastaglia FL: Search for persistent infection with poliovirus or other enteroviruses in amyotrophic lateral sclerosismotor neurone disease. Neuromuscul Disord 1995, 5:457-465

14. Vandenberghe N, Leveque N, Corcia P, Brunaud-Danel V, SalortCampana E, Besson G, Tranchant C, Clavelou P, Beaulieux F, Ecochard R, Vial C, Broussolle E, Lina B: Cerebrospinal fluid detection of enterovirus genome in ALS: a study of 242 patients and 354 controls. Amyotroph Lateral Scler 2010, 11:277-282

15. Walker MP, Schlaberg R, Hays AP, Bowser R, Lipkin WI: Absence of echovirus sequences in brain and spinal cord of amyotrophic lateral sclerosis patients. Ann Neurol 2001, 49:249-253

16. Woodall CJ, Riding MH, Graham DI, Clements GB: Sequences specific for enterovirus detected in spinal cord from patients with motor neurone disease. BMJ 1994, 308:1541-1543

17. Xue YC, Feuer R, Cashman N, Luo H: Enteroviral infection: the forgotten link to amyotrophic lateral sclerosis? Front Mol Neurosci 2018, 11:63

18. Huang HI, Shih SR: Neurotropic enterovirus infections in the central nervous system. Viruses 2015, 7:6051-6066

19. Rhoades RE, Tabor-Godwin JM, Tsueng G, Feuer R: Enterovirus infections of the central nervous system. Virology 2011, 411:288-305

20. Feuer R, Ruller CM, An N, Tabor-Godwin JM, Rhoades RE, Maciejewski S, Pagarigan RR, Cornell CT, Crocker SJ, Kiosses WB, Pham-Mitchell N, Campbell IL, Whitton JL: Viral persistence and chronic immunopathology in the adult central nervous system following Coxsackievirus infection during the neonatal period. J Virol 2009, 83:9356-9369

21. Han J, Ma XJ, Wan JF, Liu YH, Han YL, Chen C, Tian C, Gao C, Wang M, Dong XP: Long persistence of EV71 specific nucleotides in respiratory and feces samples of the patients with hand-foot-mouth disease after recovery. BMC Infect Dis 2010, 10:178

22. Julien J, Leparc-Goffart I, Lina B, Fuchs F, Foray S, Janatova I, Aymard M, Kopecka H: Postpolio syndrome: poliovirus persistence is involved in the pathogenesis. J Neurol 1999, 246:472-476

23. Fung G, Shi J, Deng H, Hou J, Wang C, Hong A, Zhang J, Jia W, Luo H: Cytoplasmic translocation, aggregation, and cleavage of TDP43 by enteroviral proteases modulate viral pathogenesis. Cell Death Differ 2015, 22:2087-2097

24. Feuer R, Mena I, Pagarigan R, Slifka MK, Whitton JL: Cell cycle status affects coxsackievirus replication, persistence, and reactivation in vitro. J Virol 2002, 76:4430-4440

25. Ruifrok AC, Johnston DA: Quantification of histochemical staining by color deconvolution. Anal Quant Cytol Histol 2001, 23:291-299

26. Ruller CM, Tabor-Godwin JM, Van Deren DA Jr, Robinson SM, Maciejewski S, Gluhm S, Gilbert PE, An N, Gude NA, Sussman MA, Whitton JL, Feuer R: Neural stem cell depletion and CNS developmental defects after enteroviral infection. Am J Pathol 2012, 180:1107-1120

27. Feuer R, Mena I, Pagarigan RR, Harkins S, Hassett DE, Whitton JL: Coxsackievirus B3 and the neonatal CNS: the roles of stem cells, developing neurons, and apoptosis in infection, viral dissemination, and disease. Am J Pathol 2003, 163:1379-1393

28. Feuer R, Pagarigan RR, Harkins S, Liu F, Hunziker IP, Whitton JL: Coxsackievirus targets proliferating neuronal progenitor cells in the neonatal CNS. J Neurosci 2005, 25:2434-2444

29. Tsueng G, Tabor-Godwin JM, Gopal A, Ruller CM, Deline S, An N, Frausto RF, Milner R, Crocker SJ, Whitton JL, Feuer R: Coxsackievirus preferentially replicates and induces cytopathic effects in undifferentiated neural progenitor cells. J Virol 2011, 85:5718-5732

30. Philips T, Robberecht W: Neuroinflammation in amyotrophic lateral sclerosis: role of glial activation in motor neuron disease. Lancet Neurol 2011, 10:253-263 
31. Eng LF, Ghirnikar RS: GFAP and astrogliosis. Brain Pathol 1994, 4: 229-237

32. Liddelow SA, Barres BA: Reactive astrocytes: production, function, and therapeutic potential. Immunity 2017, 46:957-967

33. Ahmed Z, Shaw G, Sharma VP, Yang C, McGowan E, Dickson DW: Actin-binding proteins coronin-1a and IBA-1 are effective microglial markers for immunohistochemistry. J Histochem Cytochem 2007, 55 : $687-700$

34. Paez-Gonzalez P, Asrican B, Rodriguez E, Kuo CT: Identification of distinct $\mathrm{ChAT}(+)$ neurons and activity-dependent control of postnatal SVZ neurogenesis. Nat Neurosci 2014, 17:934-942

35. Mackenzie IR, Bigio EH, Ince PG, Geser F, Neumann M, Cairns NJ, Kwong LK, Forman MS, Ravits J, Stewart H, Eisen A, McClusky L, Kretzschmar HA, Monoranu CM, Highley JR, Kirby J, Siddique T, Shaw PJ, Lee VM, Trojanowski JQ: Pathological TDP-43 distinguishes sporadic amyotrophic lateral sclerosis from amyotrophic lateral sclerosis with SOD1 mutations. Ann Neurol 2007, 61:427-434

36. Neumann M, Sampathu DM, Kwong LK, Truax AC, Micsenyi MC, Chou TT, Bruce J, Schuck T, Grossman M, Clark CM, McCluskey LF, Miller BL, Masliah E, Mackenzie IR, Feldman H, Feiden W, Kretzschmar HA, Trojanowski JQ, Lee VM: Ubiquitinated TDP-43 in frontotemporal lobar degeneration and amyotrophic lateral sclerosis. Science 2006, 314:130-133

37. Blokhuis AM, Groen EJ, Koppers $\mathrm{M}$, van den Berg LH, Pasterkamp RJ: Protein aggregation in amyotrophic lateral sclerosis. Acta Neuropathol 2013, 125:777-794
38. Yang W, Strong MJ: Widespread neuronal and glial hyperphosphorylated tau deposition in ALS with cognitive impairment. Amyotroph Lateral Scler 2012, 13:178-193

39. Lodato S, Arlotta P: Generating neuronal diversity in the mammalian cerebral cortex. Annu Rev Cell Dev Biol 2015, 31:699-720

40. Sin J, Mangale V, Thienphrapa W, Gottlieb RA, Feuer R: Recent progress in understanding coxsackievirus replication, dissemination, and pathogenesis. Virology 2015, 484:288-304

41. Rentzos M, Rombos A, Nikolaou C, Zoga M, Zouvelou V, Dimitrakopoulos A, Alexakis T, Tsoutsou A, Samakovli A, Michalopoulou M, Evdokimidis I: Interleukin-15 and interleukin-12 are elevated in serum and cerebrospinal fluid of patients with amyotrophic lateral sclerosis. Eur Neurol 2010, 63:285-290

42. Ling SC, Polymenidou M, Cleveland DW: Converging mechanisms in ALS and FTD: disrupted RNA and protein homeostasis. Neuron 2013, 79:416-438

43. Gustin KE: Inhibition of nucleo-cytoplasmic trafficking by RNA viruses: targeting the nuclear pore complex. Virus Res 2003, 95:35-44

44. Corona AK, Saulsbery HM, Corona Velazquez AF, Jackson WT: Enteroviruses remodel autophagic trafficking through regulation of host SNARE proteins to promote virus replication and cell exit. Cell Rep 2018, 22:3304-3314

45. Mohamud Y, Shi J, Qu J, Poon T, Xue YC, Deng H, Zhang J, Luo H: Enteroviral infection inhibits autophagic flux via disruption of the SNARE complex to enhance viral replication. Cell Rep 2018, 22: 3292-3303 